and Senior Lecturer, ANU Medical School, Ann Y. P. Toh Medical Student, National University Hospital, Singapore, Kua Ee Heok Head of Department and Professor of Psychological Medicine, Nationa University of Singapore

doi: $10.1192 / p b .32 .7 .275 b$

\section{Montelukast and worsening of hallucinations in paranoid schizophrenia}

A 29-year-old woman was admitted to a psychiatric in-patient unit with a history of schizophrenia. She believed that she was being raped by strangers and that people were performing witchcraft on her. She also had third-person auditory hallucinations. As the patient's symptoms did not subside with her regular antipsychotics (quetiapine $750 \mathrm{mg}$ once daily), she was considered suitable for clozapine. Later, this was contraindicated as her pulse rate was above 100 beats per minute, which was possibly related to her poor asthma control (electrocardiogram - normal QTc). The patients' antipsychotics remained unchanged, but after the review by the respiratory team, she was prescribed montelukast (10 mg once daily). In 48 hours she started to have increasing hallucinations about a spider crawling up from her abdomen to her face and biting her. She was worried that she had marks on her face and that she needed to camouflage them. She reported seeing her dead brother and talked about a 666 mark on her scalp which she saw in the mirror while combing her hair. There were no signs of delirium and the patient's MiniMental State Examination and blood tests were within normal limits.

We thought it possible that montelukast was aggravating the somatic and visual hallucinations and the medication was stopped. After 2 days the new symptoms subsided completely. Though some of the previously present psychotic symptoms were still there, the patient was less agitated than when on montelukast.

Hallucinations are rare side-effects of selective leukotriene receptor antagonists like montelukast (www.drugs.com/sfx/ montelukast-side-effects.html). The British National Formulary reports hallucinations as one of the side-effects of montelukast, but does not advise caution in schizophrenia or psychosis. Montelukast has been associated with adverse reactions such as abnormal dreaming, nightmares, hallucinations, agitation irritability and restlessness, which suggest that it can penetrate the blood-brain barrier (Price, 2000; Netherlands Pharmacovigilance Centre, 2007). A causal relation between montelukast and psychotic disorders and/or schizophrenia has not been established and is still unclear.

NETHERLANDS PHARMACOVIGILANCE CENTRE (2007) Montelukast and Depressive Symptoms. Netherlands Pharmacovigilance Centre (http:// www.lareb.nl/documents/kwb_2006_4 montel.pdf).

PRICE, D. (2000) Tolerability of montelukast. Drugs, 59 (suppl 1), 35-42.

Nithya Anandan Specialist Registrar in Psychiatry, 5 Boroughs Partnership NHS Trust, Whiston Hospita Warrington Road, Prescot, Merseyside L35 5DR, email: docnithu@yahoo.co.in,

Fade Ibitoye Consultant in Adult Psychiatry, 5 Boroughs Partnership NHS Trust, Whiston Hospitak Warrington Road, Prescot, Merseyside L35 5DR

doi: 10.1192/pb.32.7.276

\section{Questioning and answering exams}

The birth pangs of the revised College membership exam (MRCPsych) format are interesting to follow (Psychiatric Bulletin, December 2007, 31, 441-442; April 2008, 32, 152)

Though the essay and critical appraisal papers drove herds of trainees to hunt for journals, by and large it sadly remained a one-off affair. The College made a laudable attempt to cure this brief erotomania with the development of structured workplace-based assessments that qualitatively and quantitatively test the application of breaking news from the journals to clinical practice. This would ensure that the trainees use information from highimpact journals continuously and, by proxy, test the foetal findings from the laboratories in the real world of restricted resources.

The multiple choice questions, provided they are meaningfully structured to combine scientific facts and clinical practice, should test the trainees credentials or at least their recognition skills. I was distraught to find that the cut-off to pass Paper 2 was 39.8\% (personal communication) and that roughly one in three trainees had passed. This to me means that the impetus of the
College is tangential if not diametrically opposite to the focus of the trainees. This situation needs to be rectified with utmost urgency. Another hypothetical and thoroughly amusing argument is that the essay paper had enhanced the writing skills of the trainees. I am awaiting a study that will find a correlation between the trainees' scores on the essay paper and the number of their publications.

The process of changing exam patterns is an immortal amoeba. The best pseudopodia now are multiple choice questions. Whether they will breathe fresh fragrance or turn into a storm uprooting the validity of the exams will only become discernable with the passage of time.

\section{Declaration of interest}

V.R.B. is a problem-based learning tutor and is involved in the MRCPsych teaching programme at the Norfolk and Waveney Mental Health Partnership NHS Trust.

V. R. Badrakalimuthu Specialty Registrar in Old Age Psychiatry, Julian Hospital, Norfolk and Waveney Mental Health Partnership NHS Trust, Norwich, email: dr_vellingiriraja@yahoo.co.uk

doi: $10.1192 / \mathrm{pb} \cdot 32.7 .276 a$

\section{Power and glory}

Fifty-eight nominations for election to the Council of the British Medical Association have been received this year. None is a psychiatrist (although two have not specified a specialty), yet psychiatry is one of the largest fields of medicine in terms of medical staffing. Does this lack of engagement with national medical politics reflect a feeling of marginalisation within the medical workforce? Do we feel national medical representation does not reflect our needs? As a member of a regional consultants and specialists committee I find that I need to remind other members that psychiatry is not a 'minor' specialty.

In order to be seen as part of the mainstream, we need to continue to think of psychiatry as a mainstream career.

If we were organised and motivated psychiatry would be able to dominate the world of medical politics - perhaps to everyone's advantage.

Adam Moliver Consultant Old Age Psychiatrist, Charlton Lane Centre, Charlton Lane, Cheltenham GL53 9DZ, email: Adam.Moliver@glos.nhs.uk

doi: 10.1192/pb.32.7.276b 